\title{
Non-communicable disease funding
}

\author{
Luke Allen
}

The recent editorial in The Lancet Diabetes \& Endocrinology ${ }^{1}$ made a strong argument for allocating health funding in proportion to need, with an emphasis on boosting funding for non-communicable diseases (NCDs). NCDs cause $60 \%$ of disability-adjusted life-years (DALYs) and $70 \%$ of global deaths, yet they receive less than $2 \%$ of overseas development assistance for health. By contrast, HIV/AIDS accounts for $3 \%$ of global DALYS, yet receives $30 \%$ of global funds. ${ }^{2}$ Funding agencies have a range of competing priorities that determine where money is spent. Although the global burden of disease is an important factor for some, many more are focused on narrowly defined issues, and all have a fiduciary-like duty to invest in evidence-based, cost-effective strategies. By this reckoning, NCDs are a funder's nightmare: they are a poorly understood mass of conditions with loosely connected antecedents that no one actor could ever hope to cure. The most effective interventions are population strategies and multisectoral approaches that span medical, academic, and administrative domains.

Producing more data for NCDs, as The Lancet Diabetes \& Endocrinology suggests, can help to define problems more clearly by turning intractable multisectoral issues into discrete, solvable problems. ${ }^{3}$ However, several other issues ${ }^{4}$ will need attention before NCD funding increases.

NCDs are still perceived as non-infective and self-imposed in many quarters. The unhelpful non-communicable nomenclature gives nothing away to the uninitiated, and propagates the view that these conditions are not spread from person to person. The ecological and social drivers of type 2 diabetes and other NCDs will continue to be overlooked while the dominant discourse remains around individuals making healthy choices. A further issue is that the public framing of the NCD crisis has been lacklustre. The general public are unlikely to donate money to NCDs unless they understand these diseases. The weak grassroots movement might be part of the problem, with affected patients more likely to campaign for individual diseases (eg, breast cancer) than for NCDs collectively. Finally, NCDs were not included in the Millennium Development Goals and now find their solitary Sustainable Development Goal target competing with 168 others. 
There are strong human, financial, and equity-based arguments for increasing NCD funding, but it is not simply a case of shifting money from one vertical programme to another. Presenting agencies with an accurate, data-driven picture of the NCD pandemic is an opportunity to encourage funding for horizontal health-system strengthening and the promotion of primary care. I have worked in a voluntary position for the Young Professionals Chronic Disease Network, and as a consultant for the WHO Global Coordination Mechanism on NCDs.

\section{References}

1. The Lancet Diabetes \& Endocrinology. Diabetes and NCD research: meeting the needs of the patient. Lancet Diabetes Endocrinol. 2016; 4: 873

2. Institute of Health Metrics and Evaluation. Global health financing data visualization.University of Washington, Seattle2016. http://vizhub.healthdata.org/gbd-compare/ (accessed Oct 31, 2016).

3. Allen L. Non-communicable disease research. Int J Non-CommunDis (in press).

4. Allen L. Why is there no funding for non-communicable diseases? J Glob Health Perspect. 2016; (published online Oct 16.) http://jglobalhealth.org/article/why-isthere-no-funding-for-non-communicable-diseases/ 